\title{
Spontaneous inertia-gravity-wave generation by surface-intensified turbulence
}

\author{
E. Danioux ${ }^{1, a,{ }^{*}}$, J. Vanneste ${ }^{1}$, P. Klein ${ }^{2}$ and H. Sasaki ${ }^{3}$
}

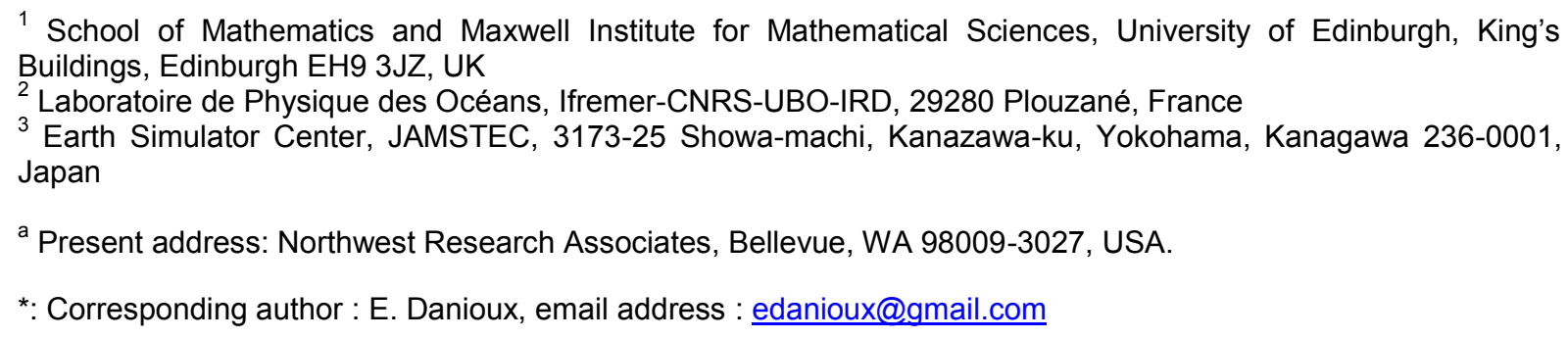

\begin{abstract}
:
The spontaneous generation of inertia-gravity waves (IGWs) by surface-intensified, nearly balanced motion is examined using a high-resolution simulation of the primitive equations in an idealized oceanic configuration. At large scale and mesoscale, the dynamics, which is driven by baroclinic instability near the surface, is balanced and qualitatively well described by the surface quasigeostrophic model. This however predicts an increase of the Rossby number with decreasing spatial scales and, hence, a breakdown of balance at small scale; the generation of IGWs is a consequence of this breakdown. The wave field is analysed away from the surface, at depths where the associated vertical velocities are of the same order as those associated with the balanced motion. Quasigeostrophic relations, the omega equation in particular, prove sufficient to separate the IGWs from the balanced contribution to the motion. A spectral analysis indicates that the wave energy is localized around dispersion relation for free IGWs, and decays only slowly as the frequency and horizontal wavenumber increase. The IGW generation is highly intermittent in time and space: localized wavepackets are emitted when thin filaments in the surface density are formed by straining, leading to large vertical vorticity and correspondingly large Rossby numbers. At depth, the IGW field is the result of a number of generation events; away from the generation sites it takes the form of a relatively homogeneous, apparently random wave field. The energy of the IGW field generated spontaneously is estimated and found to be several orders of magnitude smaller than the typical IGW energy in the ocean.
\end{abstract}

Keywords: internal waves ; quasi-geostrophic flows ; wave-turbulence interactions

\section{Introduction}

A fundamental feature of geophysical fluid dynamics, relevant to both the atmosphere and ocean, is the separation between balanced motion and inertia-gravity waves (IGWs). In many regions, the two types of motion are well separated, with the slow, balanced motion largely unaffected by the much faster IGWs. This separation, which stems from the large frequency gap between the two types of motion and is estimated by the (small) Rossby number, is well understood. The separation is not complete, however; as a result, balanced motion constantly leaks some energy to IGWs, thus providing a source of IGW generation through the mechanism known as spontaneous generation. This mechanism is of interest in particular because it potentially excites IGWs across the whole spectrum of wavelengths and frequencies. This contrasts with other sources, such as tidal and wind forcing in the ocean, or topographic and convective forcing in the atmosphere, which have a determined frequency. 
In recent years, spontaneous generation has been demonstrated in a number of numerical simulations (e.g., O'Sullivan \& Dunkerton 1995; Zhang 2004; Viudez \& Dritschel 2006; Plougonven \& Snyder 2005, 2007; Snyder et al. 2007) and in idealised models (e.g., Vanneste \& Yavneh 2004; Ólafsdóttir et al. 2008). Theoretical arguments suggest that this generation is exceedingly small when the Rossby number is small - exponentially so, as asymptotic results indicate (Vanneste 2008b, and references therein). Non-negligible IGW generation is therefore expected only in regions where the Rossby number is of order one or larger. In the absence of direct external forcing, large Rossby numbers typically do not emerge in the interior of geophysical fluids: flows that are balanced initially remain so. This is true even in the presence of turbulence because the steep energy spectra of the (forward, enstrophy) cascade of balanced turbulence is associated with Rossby numbers that are essentially scale-independent. However, the situation changes radically when horizontal boundaries such as the ocean or earth's surface, or the tropopause are taken into account. In this case, the evolution of the surface (potential) temperature which, together with the interior potential vorticity, controls the balanced dynamics, leads to shallow energy spectra, vorticity intensification and hence large local Rossby numbers at the surface. This is most easily demonstrated using the surface-quasi-geostrophic (SQG) model (e.g. Held et al. 1995). Indeed simple scaling arguments based on the surface energy spectrum $E\left(k_{\mathrm{h}}\right) \sim k_{\mathrm{h}}^{-5 / 3}$ predicted in SQG suggest that the Rossby number scales with the horizontal wavenumber as Ro $\sim k_{\mathrm{h}}^{2 / 3}$ (see Blumen 1978; Juckes 1994, Eq. (7.14)), so that large Rossby numbers always appear at small enough horizontal scales in nearsurface flows even though they are balanced at large scales. Therefore, as Juckes (1994) argues, balance breaks down at some stage. IGW generation can be expected at some point in this process.

These scaling arguments are substantiated by process studies which have identified frontogenesis as a mechanism for IGW generation by surface-intensified flows. The classical two-dimensional models of frontogenesis driven by vertical shear (Williams 1967) or horizontal strain (Hoskins \& Bretherton 1972), which in the semi-geostrophic approximation predict a finite-time singularity, have been examined using two-dimensional primitive-equation simulations (Snyder et al. 1993; Griffiths \& Reeder 1996; Reeder \& Griffiths 1996, and references therein). These studies demonstrate that the breakdown of the (semi-geostrophic) balance in these flows is accompanied by the emission of IGWs whose amplitudes depend strongly on the speed of the frontogenesis evolution. This mechanism has also been found in three-dimensional simulations of baroclinic instability where surface fronts develop (Zhang 2004; Plougonven \& Snyder 2007).

While most of the work on the breakdown of balance near horizontal boundaries was motivated by applications to the atmosphere, the importance of near-surface, SQG-like motion in the ocean is increasingly recognised (e.g. Lapeyre \& Klein 2006). In particular, the formation of fronts that characterise surface motion, and their subsequent instability in regimes far from geostrophy have been identified as sources of submesoscale motion (Capet et al. 2008a,b,c; McWilliams et al. 2009), along with possible implications for the the energy balance in the ocean (Molemaker et al. 2010).

In the present paper, we also consider the oceanic context, but with a different focus which concentrates on IGW generation. The main aim is to demonstrate that the argument put forward by Juckes (1994) applies to the ocean and that IGWs are generated spontaneously by what can be described in the first approximation as SQG turbulence. While the generation process involved is similar to the frontal emission of Snyder et al. (1993) and Griffiths \& Reeder (1996), our focus is on the global behaviour of a complex, turbulent, three-dimensional flow as a wave emitter rather than on single wave emis- 
sion events. We pay particular attention to the spectral characteristics of the IGW field. Specifically, we analyse a high-resolution simulation of an ocean jet driven by baroclinic instability into a regime of statistically steady, surface-intensified turbulence, and examine how this turbulence spontaneously radiates IGWs which propagate into the ocean's interior. The IGW signal is weak compared to the balanced signal, and best inferred at depth since the balanced signal decays with depth (exponentially so in SQG). We use diagnostic tools based on quasi-geostrophic theory, the omega equation in particular, in order to separate IGWs and balanced motion. The analysis shows that spontaneous generation is highly intermittent both in space and time: wavepackets are produced by a number of generation events which we associate with the formation of thin filaments of surface density (here equivalent to potential temperature), with strong vertical vorticity, which is produced by straining. Although the emission of inertia-gravity wavepackets is highly intermittent, their subsequent propagation and dispersion leads to what appears as a random, almost homogeneous IGW field at depth. A spectral analysis of this field confirms that it consists of free IGWs obeying the dispersion relation. Our results support the argument that the cascade to small-scale inherent to surface motion leads to balance breakdown and IGWs generation. However, an estimation of the energy associated with these spontaneously emitted waves indicates that it is orders of magnitude smaller than the typical energy for (wind- and tidally-driven) internal waves.

The plan of the paper is as follows. We briefly describe the numerical model used and the set-up of the simulation in $\S 2$. Section 3 is devoted to the diagnostic tools necessary to separate IGWs from the balanced motion. These use the omega equation and another quasi-geostrophic balance relation, together with a specific energy-minimising projection. The IGW field obtained in the simulation is analysed in $\S 4$. We first examine the physical and spectral characteristics of the entire wave field, estimate its energy, before describing a typical wave-generation event. The paper concludes with a discussion in $\S 5$.

\section{Model set-up}

We analyse a high-resolution simulation of ocean turbulence modelled by the primitive equations under the Boussineq and hydrostatic approximations. The numerical model and parameter set-up employed for this simulation are similar to those in Klein et al. (2008). We describe them briefly and refer the reader to this paper for more detail.

The numerical model (ROMS, Shchepetkin \& McWilliams 2005) is a $\sigma$-coordinate model used in a periodic channel configuration, with free-slip boundary conditions at northern and southern walls. The domain size is $500 \times 2000 \mathrm{~km}$ in the horizontal, with a depth of $4 \mathrm{~km}$. The numerical resolution is $2 \times 2 \mathrm{~km}$ in the horizontal and 100 levels in the vertical (the vertical grid spacing ranges from $3 \mathrm{~m}$ near the surface to $200 \mathrm{~m}$ near the bottom). Vertical mixing in the interior is represented by a K-profile parameterization (KPP, Large et al. 1994). The numerical dissipation of the model is sufficient for stability without resorting to explicit viscous damping. Salinity is taken as constant, so that temperature anomalies are proportional to density anomalies. There is no atmospheric forcing.

An energetic turbulent mesoscale and submesoscale eddy field is generated through the baroclinic instability of a balanced, surface-intensified, westerly zonal flow centred in the middle of the channel. The parameter settings for this flow resemble those used by Karsten et al. (2002), Rivière et al. (2004) and Lapeyre \& Klein (2006). The initial vertical profile of the mean Brunt-Väisälä frequency (Fig. 1) represents a main thermocline located at a depth around $600 \mathrm{~m}$, corresponding to a first Rossby radius of deformation of approximately $25 \mathrm{~km}$. No surface mixed layer is initially present. The zonal flow is 


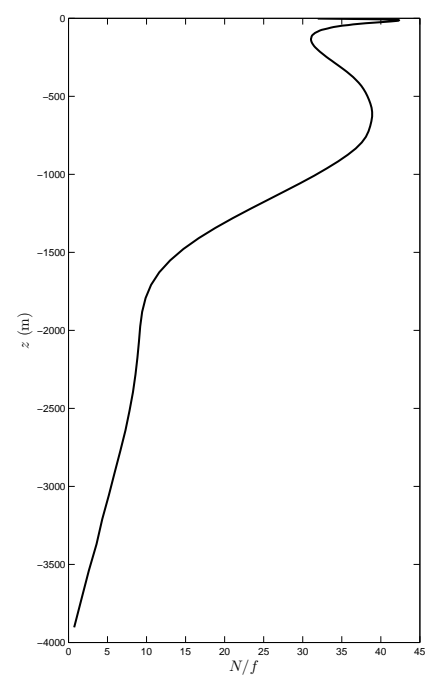

FigURE 1. Horizontally averaged profile of the ratio of the Brunt-Väisälä frequency to the Coriolis frequency in the simulation.

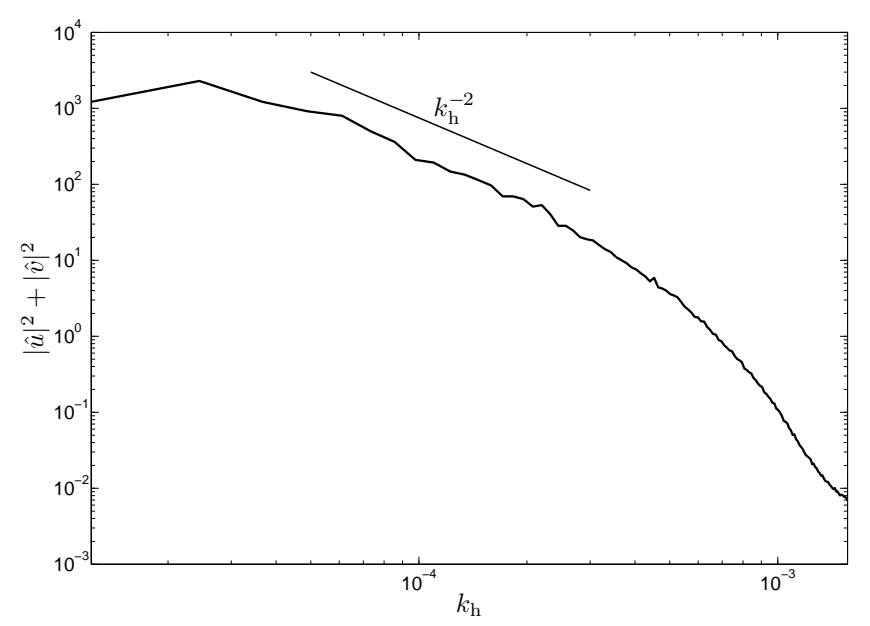

FiguRE 2. Horizontal wavenumber spectrum of the surface kinetic energy.

maintained throughout the simulation using a relaxation of the zonally averaged velocity and density fields to the initial state. The relaxation time is chosen as 50 days. While maintaining the zonal-mean flow, this forcing does not damp the eddies, leading to a vigorous turbulent eddy field.

After a spinup of 350 days, the total kinetic energy (integrated over the whole domain) has saturated, and a statistically steady state is reached. The results described in this paper concern the flow well after this spinup period. The surface energy spectrum, shown in Fig. 2, approximately displays a $k_{\mathrm{h}}^{-2}$ dependence for lengthscales ranging from $20 \mathrm{~km}$ to $130 \mathrm{~km}$, and a $k_{\mathrm{h}}^{-3}$ dependence for lengthscales ranging from $10 \mathrm{~km}$ to $20 \mathrm{~km}$. This $k_{\mathrm{h}}^{-2}$ spectrum is substantially steeper than the SQG prediction $E\left(k_{\mathrm{h}}\right) \sim k_{\mathrm{h}}^{-5 / 3}$, but in agreement with the results of Lapeyre \& Klein (2006). We refer the reader to that paper for a detailed discussion of the applicability and limitations of SQG as a model of the near-surface flow. From our viewpoint, what it important is that this steeper spectrum 
does not invalidate the argument about Rossby numbers increasing with $k_{\mathrm{h}}$ mentioned in $\S 1$ : using the frequency estimate $\omega \sim\left(\int^{k_{\mathrm{h}}}{k^{\prime}}^{2} E\left(k^{\prime}\right) d k^{\prime}\right)^{1 / 2}$ (Kraichnan 1971) with $E\left(k_{\mathrm{h}}\right) \sim k_{\mathrm{h}}^{-2}$ yields Ro $\sim \omega / f \sim k_{\mathrm{h}}^{1 / 2}$.

The surface vorticity typically varies between $-f$ and $4 f$, where $f$ is the Coriolis frequency. These large values, which arise locally from the straining of small-scale density filaments, are associated with the generation of IGWs. The asymmetry between cyclonic and anticyclonic vorticity is marked; as a result, IGW generation is largely limited to cyclonic flow features. Note that while the mesoscale field obtained is strongly energetic, there is substantially less activity at the smallest scales than in the simulations of Capet et al. (2008b) and in particular no evidence of submesoscale instabilities, presumably as a result of our lower horizontal resolution $(2 \mathrm{~km}$ vs. $750 \mathrm{~m}$ ) and larger numerical viscosity.

\section{Balanced motion and inertia-gravity waves}

Our aim is to examine the IGWs that are generated spontaneously by the near-surface, balanced turbulence and propagate into the deep ocean. The distinction between balanced motion and IGWs, or more generally unbalanced motion, is clear in the limit of small Rossby number Ro $\ll 1$. In this limit, it is possible to define balance relations which relate all dynamical variables to a single one, often potential vorticity. Geometrically, this is often thought of as the definition of slow manifolds which are approximately invariant: nearly balanced motion evolves in the neighbourhood of these manifolds but slowly drifts away as a result of spontaneous generation of IGWs. Balanced relations are most easily constructed systematically using asymptotic series expansions in powers of Ro; this leads to a hierarchy of relations of higher-and-higher accuracy, starting with geostrophic balance (Warn et al. 1995; Vanneste 2008b). Unless the asymptotic expansions are carried out to optimal truncation, the separation between balanced and unbalanced motion is always ambiguous: the difference between the full dynamical fields and those of the corresponding balanced state obtained by projection on a slow manifold results from the limited accuracy of the balance relation used as well as from the presence of genuine unbalanced motion.

In what follows, we will only use the leading-order, geostrophic balance. This is sufficient for our purpose because we concentrate on the fields at depth: in qualitative agreement with SQG theory, the intensity of the turbulence in the flow analysed decays rapidly with depth while its typical horizontal scales increase. This leads to Rossby numbers at depth that are much smaller than at the surface; for instance using the ratio of relative to planetary vorticity gives on average $R o<0.06$ at $2500 \mathrm{~m}$. In addition, the characteristics of the unbalanced motion away from the region of generation are different from those of the balanced motion. They consist of small-scale IGWs with a clear wavenumber-frequency signature. We now discuss the methods we use to extract this IGW signal using the geostrophic balance relation.

\subsection{Vertical velocity}

Let us consider the separation of the dynamical fields between a geostrophic, a balanced ageostrophic and unbalanced contribution. By balanced ageostrophic, we mean the corrections to geostrophic balance that are obtained using higher-order balance relations which slave all the fields to a single one, say the potential vorticity. As mentioned, these relations can be obtained systematically using asymptotic expansions in powers of the Rossby number (Warn et al. 1995); since they diverge, the expansions must be truncated; conceptually (if not practically), it is useful to think of the truncation as being an optimal 
one, near the smallest term (Bender \& Orszag 1999), so that the remainder is dominated by unbalanced motion (Vanneste 2008b, a; Temam \& Wirosoetisno 2011).

The decomposition of the horizontal and vertical nondimensionalized velocities gives terms of the following orders of magnitudes:

$$
u=\underbrace{u_{\mathrm{g}}}_{O(1)}+\underbrace{u_{\mathrm{bag}}}_{O(\mathrm{Ro})}+\underbrace{u_{\mathrm{unb}}}_{O(\alpha)} \text { and } w=\underbrace{w_{\mathrm{bag}}}_{O(\mathrm{Ro})}+\underbrace{w_{\mathrm{unb}}}_{O(\alpha)},
$$

where $\alpha$ is the amplitude of the unbalanced motion which we aim to determine. Most fields, the density in particular, obey a scaling comparable to that of the horizontal velocity $u$. This makes it clear that the vertical velocity $w$ is better suited for the identification of IGWs than these other fields (the divergence of the horizontal velocity field is similar in this respect). The analysis below suggests that $\alpha$ and Ro have comparable orders of magnitude in the bottom half of our simulation, so that the unbalanced part of $w$ can be detected in the total field. Note however that this is based on a local Ro whereas the validity of balance relations, which involve non-local operators, in principle requires that Ro be uniformly small - an assumption that is clearly not satisfied in the flow we consider.

Although IGWs can be identified in the total vertical velocity $w$, mainly thanks to their distinctive spatial structure (see $\S 4$ ), it is useful to use a balance relation in order to diagnose $w_{\text {bag }}$ and better isolate the IGW contribution $w_{\text {unb }}$. This relation is provided by the quasi-geostrophic omega equation (Hoskins et al. 1978)

$$
\nabla_{\mathrm{h}}^{2} w_{\mathrm{qg}}+\frac{f^{2}}{N^{2}} \frac{\partial^{2} w_{\mathrm{qg}}}{\partial z^{2}}=\frac{2 g}{\rho_{0} N^{2}} \nabla_{\mathrm{h}} \cdot \mathbf{Q},
$$

where $\nabla_{\mathbf{h}}=\left(\partial_{x}, \partial_{y}\right)$ denotes the horizontal gradient, and

$$
\mathbf{Q}=\left(\nabla_{\mathrm{h}} \mathbf{u}_{\mathrm{h}}\right)^{\mathrm{T}} \cdot \nabla_{\mathrm{h}} \rho,
$$

with $\mathbf{u}_{\mathrm{h}}=(u, v)$ the horizontal velocity. Eq. (3.1) is solved with the boundary conditions $w_{\mathrm{qg}}(z=0)=w_{\mathrm{qg}}(z=-H)=0$. The geostrophic approximation to the vertical velocity that is obtained, $w_{\mathrm{qg}}$, is the leading order of $w_{\mathrm{bag}}: w_{\mathrm{bag}}=w_{\mathrm{qg}}+O\left(\mathrm{Ro}^{2}\right)$. Note that, in quasi-geostrophic theory, $\mathbf{Q}$ is defined in terms of the geostrophic approximation to $\mathbf{u}_{\mathrm{h}}$ and $\rho$; instead, we use the full fields since this provide an estimate of $w_{\text {bag }}$ with the same formal accuracy. The use of the quasi-geostrophic omega equation (3.1), compared to more sophisticated balance relations (e.g. Muraki et al. 1999) or approaches taking into account dissipation in the surface mixed layer (Nagai et al. 2006), is justified a posteriori by the efficiency of our method (see Figs. 3 and 4).

For the results reported in section 4, we solve (3.1) on the ROMS grid using the freeaccess mudpack solver (multigrid software for elliptic partial differential equations, see Adams (1989)). Once $w_{\mathrm{qg}}$ is obtained, the difference $w-w_{\mathrm{qg}}=w_{\mathrm{unb}}+O\left(\mathrm{Ro}^{2}\right)$ gives an estimate for the IGW contribution to the vertical velocity.

\subsection{Horizontal velocity}

For comparison, it is also useful to estimate the IGW contribution to dynamical fields other than $w$. If a geostrophic approximation is computed, say for the zonal velocity $u$, then the IGWs may be identifiable by considering $u-u_{\mathrm{g}}=u_{\mathrm{unb}}+O(\mathrm{Ro})$. The diagnostic of balanced fields from data raises an issue which appears to be often overlooked, the need for a projection: a balance relation, geostrophy in our case, defines a slow manifold, but it does not give a unique means of projecting arbitrary data on this slow manifold.

A possible projection consists in insisting that the potential vorticity be the same 
in the unprojected state (off the slow manifold) and the projected state (on the slow manifold). This particular projection does not have a strong motivation, and it does not necessarily make obvious which potential vorticity (Rossby-Ertel, quasi-geostrophic, linearised) should be chosen. Here we adopt a somewhat different approach: for given velocity and density fields $\mathbf{u}$ and $\rho$, we seek the nearest geostrophic state

$$
\left(\mathbf{u}_{\mathrm{g}}, \rho_{g}\right)=\left(\nabla_{\mathrm{h}}^{\perp} \psi,-\frac{\rho_{0} f_{0}}{g} \frac{\partial \psi}{\partial z}\right), \quad \text { with } \nabla_{\mathrm{h}}^{\perp}=\left(-\partial_{y}, \partial_{x}\right),
$$

where 'nearest' is understood in the sense of some norm. The choice of norm is arbitrary; we choose a norm based on the average energy per unit volume

$$
\mathcal{E}=\frac{\rho_{0}}{2 V} \iiint\left[u^{2}+v^{2}+\left(\frac{g \rho}{N \rho_{0}}\right)^{2}\right] d x d y d z,
$$

where $V$ is the domain volume, which is conserved for the primitive equations in the absence of forcing and dissipation and for suitable boundary conditions (e.g. no normal velocity). Specifically, we project any state on the geostrophic state (3.2) by finding the streamfunction $\psi$ which achieves

$$
\min _{\psi}\left(\frac{\rho_{0}}{2 V} \iiint\left[\left|\mathbf{u}-\mathbf{u}_{\mathbf{g}}\right|^{2}+\left(\frac{g}{N \rho_{0}}\right)^{2}\left(\rho-\rho_{g}\right)^{2}\right] d x d y d z\right) .
$$

For the boundary conditions $\rho_{g}=\rho$ at $z=0,-H$ and $\mathbf{u}_{\mathrm{g}}=\mathbf{u}_{\mathrm{h}}$ on the lateral boundaries, a simple variational calculation shows that this minimum is reached when $\psi$ solves the equation

$$
\nabla_{\mathrm{h}}^{2} \psi+\frac{\partial}{\partial z}\left(\frac{f^{2}}{N^{2}} \frac{\partial \psi}{\partial z}\right)=\hat{\mathbf{z}} \cdot\left(\nabla_{\mathrm{h}} \times \mathbf{u}_{\mathrm{h}}\right)-\frac{g f}{\rho_{0}} \frac{\partial}{\partial z}\left(\frac{\rho}{N^{2}}\right),
$$

where $\hat{\mathbf{z}}$ denotes a vertical unit vector.

The left hand side of (3.5) is the usual quasi-geostrophic potential vorticity, a quantity that is conserved by the advection in the quasi-geostrophic approximation. The righthand side is neither the total potential vorticity nor the linearised vorticity divided by $N^{2}$ (in both cases the factor $N^{-2}$ would be undifferentiated); rather it resembles the quasi-geostrophic potential vorticity, with the total velocity and density replacing their quasi-geostrophic approximations $\nabla^{\perp} \psi$ and $-f \rho_{0} \partial_{z} \psi / g$, respectively. We solve (3.5), like (3.1), on the ROMS grid using the mudpack solver.

\section{3. $I G W$ energy}

The discussion above indicates that for a similar computational effort (the solution of a three-dimensional linear elliptic equation) we can obtain either the sum $w_{\text {unb }}+O\left(\mathrm{Ro}^{2}\right)$ or the sum $\mathbf{u}_{\mathrm{unb}}+O(\mathrm{Ro})$, where the error terms consist of the balanced part of the flow not captured by geostrophic balance. It is clear, therefore, that it is advantageous to express quantities such as the unbalanced energy in terms of $w$ alone when possible.

One way to do so is to project the dynamical fields on suitable vertical normal modes and exploit results of linear wave theory, namely the IGW dispersion and polarisation relations, to relate all dynamical fields to the projection of $w$.

Let us define the functions $F_{n}$ as eigenfunctions of the Sturm-Liouville problem

$$
\partial_{z}\left(\frac{f^{2}}{N^{2}} \partial_{z} F_{n}\right)=-\frac{1}{r_{n}^{2}} F_{n}, \quad \partial_{z} F_{n}=0 \text { at } z=0,-H,
$$

where the deformation radii $r_{n}$ are the eigenvalues (e.g. Gill 1982). We define the addi- 
tional functions $G_{n}$ and $H_{n}$ by

$$
G_{n}(z)=r_{n} f \partial_{z} F_{n}, \quad H_{n}(z)=\int_{z}^{0} F_{n}\left(z^{\prime}\right) d z^{\prime}
$$

(see Danioux et al. (2008) and Danioux \& Klein (2008)). It is easy to verify that these functions are orthogonal in the sense that

$$
\int_{-H}^{0} F_{n} F_{m} d z=\delta_{m, n}, \quad \int_{-H}^{0} N^{-2} G_{n} G_{m} d z=\delta_{m, n}, \quad \int_{-H}^{0} N^{2} H_{n} H_{m} d z=f^{2} r_{n}^{2} \delta_{m, n} .
$$

The $F_{n}, G_{n}$ and $H_{n}$ can be used to project the dynamical fields according to

$$
(u, v)=\sum_{n=1}^{\infty}\left(u_{n}, v_{n}\right) F_{n}, \quad w=\sum_{n=1}^{\infty} w_{n} H_{n}, \quad \rho=\sum_{n=1}^{\infty} \rho_{n} G_{n} .
$$

Here $u_{n}, v_{n}, w_{n}$ and $\rho_{n}$ are functions of $x, y$ and $t$. Their Fourier transforms with respect to these three independent variables, denoted by a hat, satisfy

$$
u_{n}=\iiint \hat{u}_{n}(k, l, \omega) e^{i(k x+l y-\omega t)} d k d l d \omega
$$

and similar. Introducing (3.7)-(3.8) into the linearised hydrostatic-Boussinesq equations leads to the dispersion relation

$$
\omega^{2}=f^{2}+f^{2} r_{n}^{2}\left(k^{2}+l^{2}\right),
$$

for each mode $n$ with frequency $\omega$ and horizontal wavenumbers $(k, l)$. The corresponding polarisation relations read

$$
\begin{aligned}
& \hat{u}_{n}=\left(\frac{f l}{\omega\left(k^{2}+l^{2}\right)}-i \frac{k}{k^{2}+l^{2}}\right) \hat{w}_{n}, \\
& \hat{v}_{n}=-\left(\frac{f k}{\omega\left(k^{2}+l^{2}\right)}+i \frac{l}{k^{2}+l^{2}}\right) \hat{w}_{n}, \\
& \hat{\rho}_{n}=i \frac{\rho_{0} r_{n} f}{g \omega} \hat{w}_{n} .
\end{aligned}
$$

Note that, for a given mode $n$ and horizontal wavenumber $(k, l)$, the energy is concentrated on two opposite frequencies solution of (3.9).

Using equations (3.9) and (3.10), the vertical profile of the energy (3.3) averaged in time becomes

$$
\mathcal{E}_{z}(z)=\frac{\rho_{0}}{2 V T} \sum_{n} \iiint\left(\frac{\omega^{2}+f^{2}}{\omega^{2}\left(k^{2}+l^{2}\right)} F_{n}^{2}(z)+\frac{\omega^{2}-f^{2}}{\omega^{2}\left(k^{2}+l^{2}\right)} \frac{G_{n}^{2}(z)}{N^{2}(z)}\right)\left|\hat{w}_{n}\right|^{2} d k d l d \omega,
$$

assuming that the domain size and duration $T$ of the simulation are much larger than the wavelengths and periods of the energy-containing IGWs.

The first and second terms on the right-hand side of (3.11) represent the kinetic and potential energies respectively. One can integrate this equation over the depth of the domain using the orthogonality relations (3.6), and find the following equation for the total energy per volume unit:

$$
\mathcal{E}=\int_{-H}^{0} \mathcal{E}_{z}(z) d z=\frac{\rho_{0}}{V T} \sum_{n} \iiint \frac{\left|\hat{w}_{n}\right|^{2}}{k^{2}+l^{2}} d k d l d \omega .
$$



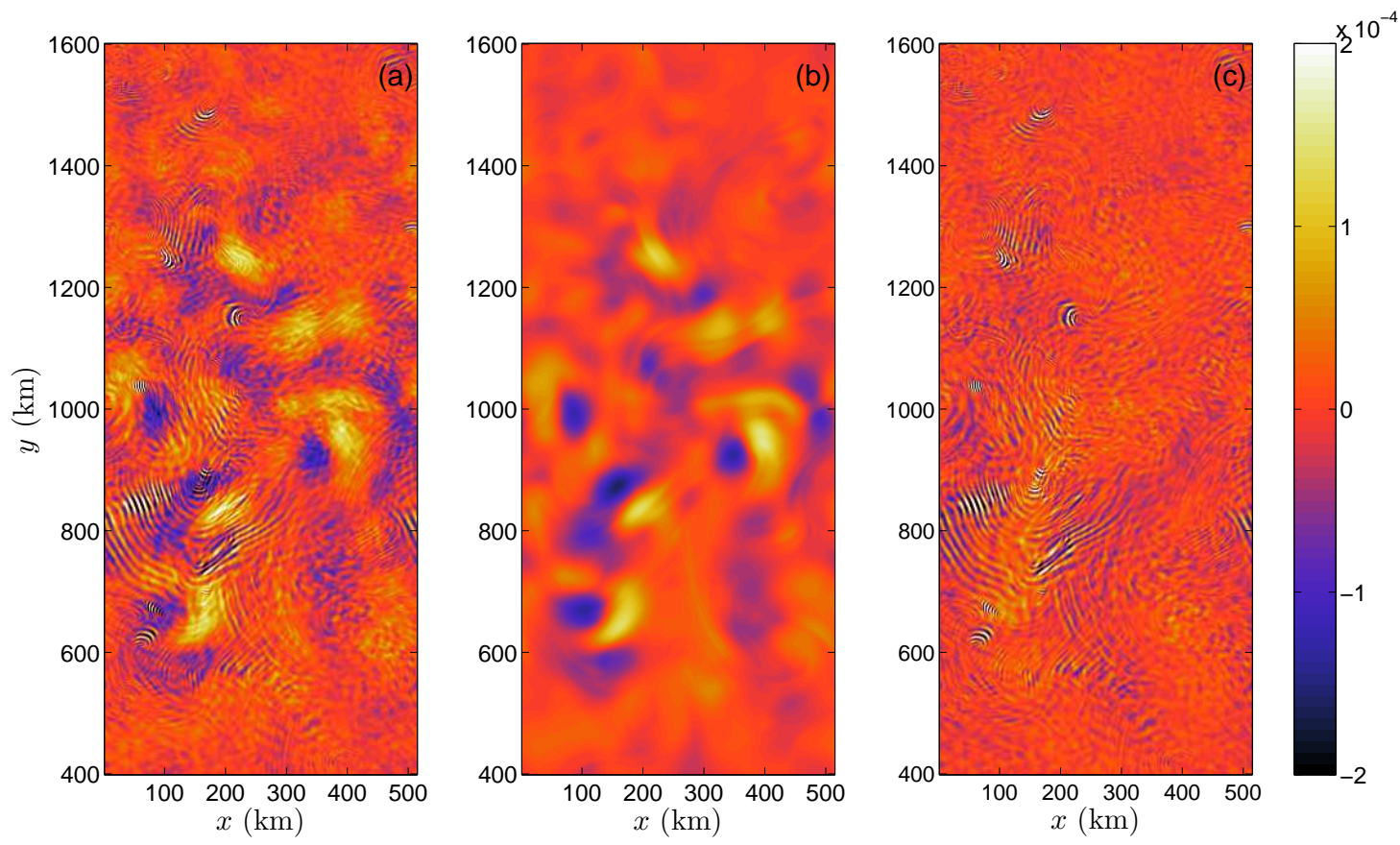

Figure 3. Vertical velocity (in $\mathrm{m} \mathrm{s}^{-1}$ ) at $z=-2500 \mathrm{~m}$ : (a) total velocity $w$; (b) quasigeostrophic approximation $w_{\text {qg }}$ obtained by solving the omega equation; (c) unbalanced contribution $w_{\mathrm{unb}}=w-w_{\mathrm{qg}}$. Note that absolute values larger than $2 \cdot 10^{-4} \mathrm{~m} \mathrm{~s}^{-1}$ may occur locally.

This formula is useful to estimate the IGW contribution to the total energy from the unbalanced vertical velocity only.

\section{Results}

\subsection{Extraction of the wave signal}

We apply the two methods of extraction of the IGW vertical and horizontal velocities described in $\S \S 3.1-3.2$ to our numerical simulation and examine the results both in the physical and the frequency-wavenumber domains. We use model data sampled every 1.5 hour; this high temporal resolution and the relatively long sample used (120 hours) make it possible to resolve frequencies in the range $0.15 f$ to $6 f$.

A typical snapshot of the vertical velocity field at $z=-2500 \mathrm{~m}$ is shown in Fig. 3: the total field $w$ (Fig. 3a) is compared with the solution $w_{\text {qg }}$ of the omega equation (Fig. 3b) and with the unbalanced contribution $w_{\mathrm{unb}}=w-w_{\mathrm{qg}}$ (Fig. 3c). At this depth, the total vertical velocity appears to be a superposition of a large-scale balanced motion and a small-scale unbalanced motion. Both types of motion have similar amplitudes, with a r.m.s. value of about $3 \cdot 10^{-5} \mathrm{~m} \mathrm{~s}^{-1}$. However, the unbalanced velocity displays much stronger extrema with values reaching $10^{-3} \mathrm{~m} \mathrm{~s}^{-1}$, while the balanced velocity does not exceed $2 \cdot 10^{-4} \mathrm{~m} \mathrm{~s}^{-1}$. The depth of $2500 \mathrm{~m}$ is intermediate in our simulation: below $2500 \mathrm{~m}$ the unbalanced vertical velocities dominate the balanced ones (the ratio of their r.m.s. tends to 4 close to the bottom), while above $2500 \mathrm{~m}$ balanced vertical velocities dominate (the ratio of their r.m.s. is 0.25 at $z=-1000 \mathrm{~m}$ for instance). The comparison of the three panels of Fig. 3 highlights the effectiveness of the omega equation as a tool 
to separate the balanced and unbalanced components of the flow: the balanced $w_{\mathrm{qg}}$ is free from traces of the small-scale IGWs, while the unbalanced $w-w_{\mathrm{qg}}$ is dominated by IGWs, with only a weak remnant of the large-scale balanced motion.

The overall picture of the unbalanced vertical velocity at $2500 \mathrm{~m}$ is one of roughly (horizontally) isotropic interfering waves. The amplitude of these waves is largely homogeneous, with r.m.s. values of $2 \cdot 10^{-5} \mathrm{~m} \mathrm{~s}^{-1}$ on most of the domain. However, at any one time, a few regions display a much stronger wave activity, characterized by much higher local r.m.s. values (up to $5 \cdot 10^{-4} \mathrm{~m} \mathrm{~s}^{-1}$ ). At the time chosen for Fig. 3, regions of this type appear for instance around $(x, y)=(210 \mathrm{~km}, 1150 \mathrm{~km})$ or $(x, y)=(70 \mathrm{~km}, 630 \mathrm{~km})$. The strong curvature of the crests and troughs in these regions suggest that the waves there have just been emitted. Another interesting region extends from $x=0 \mathrm{~km}$ to $250 \mathrm{~km}$ and $y=700 \mathrm{~km}$ to $900 \mathrm{~km}$. The shape of the waves in this region strongly suggest a prior emission around $(x, y)=(80 \mathrm{~km}, 450 \mathrm{~km})$; this has been verified on plots of the three vertical velocities at earlier times (not shown).

The picture that emerges from the simulation, especially when examining animations of the vertical velocity data, is of a generation of IGWs that is highly intermittent in space and time. After each generation event, the waves emitted propagate, disperse, are refracted and interfere to create a complex time-dependent wave field.

A view of the unbalanced motion complementary to that given by the representation of the field in physical space is provided by power spectra in the horizontal wavenumberfrequency domain. Fig. 4 displays several such spectra: the left panels show the spectra of the vertical velocities $w, w_{\mathrm{qg}}$ and $w-w_{\mathrm{qg}}$ (Figs. 4a-c); the right panels show the kinetic energy spectra corresponding to the total horizontal velocity, the balanced horizontal velocity obtained by solving (3.5) and the unbalanced horizontal velocity derived by substraction (Figs. $4 \mathrm{~d}-\mathrm{f}$ ). These spectra have been obtained at a depth $z=-2500 \mathrm{~m}$ by first calculating the horizontal Fourier transform $\tilde{\phi}(k, l, z, t)$ of each variable $\phi(x, y, z, t)$ (where $\phi$ is the total, balanced and unbalanced vertical or horizontal velocity), then Fourier-transforming in time to obtain the horizontal wavenumber-frequency transform $\hat{\phi}(k, l, z, \omega)$ and finally summing the values of $|\hat{\phi}(k, l, z, \omega)|^{2}$ over the wavenumbers $(k, l)$ such that $\sqrt{k^{2}+l^{2}} \in\left[k_{\mathrm{h}}, k_{\mathrm{h}}+d k_{\mathrm{h}}\right]$.

The total vertical velocity spectrum (Fig. 4a) displays two distinct energetic regions: the first region is characterized by low-frequencies $(\omega \lesssim f / 2)$ and relatively large scales $\left(k_{\mathrm{h}} \lesssim 1 \cdot 10^{-4} \mathrm{~m}^{-1}\right)$, while the second region is characterized by high frequencies $(\omega \gtrsim f)$ and small scales $\left(k_{h} \gtrsim 1 \cdot 10^{-4} \mathrm{~m}^{-1}\right)$. The first region is associated with the balanced motion, as is confirmed by the spectrum of the balanced velocity $w_{\mathrm{qg}}$ (Fig. $4 \mathrm{~b}$ ); the second region is associated with the unbalanced motion (Fig. 4c). The power integrated over these two regions in the $\left(\omega, k_{h}\right)$ spectrum has been computed and give similar values (about $3 \cdot 10^{-5} \mathrm{~m} \mathrm{~s}^{-1}$ ), consistent with the r.m.s. associated with the two types of motion in physical space. Note that although most of the power of the unbalanced vertical velocity in Fig. 4c is contained at frequencies higher than $f$, some remains at lower frequencies because the omega equation provides only a first-order approximation to the balanced velocity. Note also that the balanced velocity spectrum is absolutely depleted of energy in the region $\left(\omega \gtrsim f, k_{\mathrm{h}} \gtrsim 2 \cdot 10^{-4} \mathrm{~m}^{-1}\right)$ : this shows that the omega equation efficiently filters out IGWs.

The dispersion relation for the first few IGW modes, given by $\omega^{2}=f^{2}+f^{2} r_{n}^{2} k_{h}^{2}$ (see (3.9)) is also plotted in Fig. 4. The unbalanced vertical power (Fig. 4c) follows the dispersion curves well, confirming that the unbalanced activity consists mainly of free linear IGWs. A striking feature in the figure is that the vertical-velocity spectrum is almost constant along the dispersion curves; in particular there is no marked decay as the 

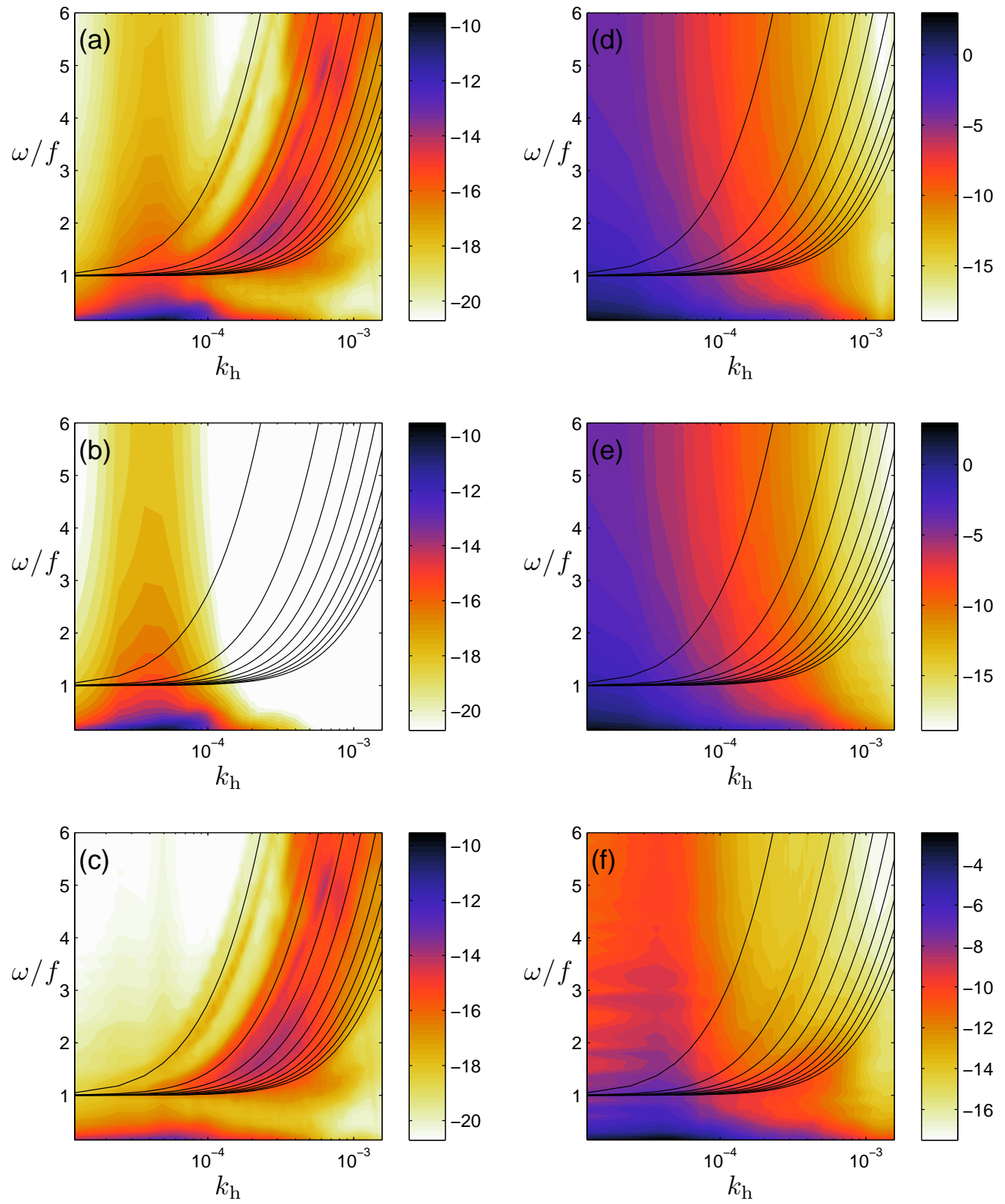

FigURE 4. Frequency-horizontal wavenumber spectrum (log-scale) at $z=-2500 \mathrm{~m}$. Left panels: vertical velocity, $w(\mathrm{a}), w_{\text {qg }}$ (b) and $w_{\text {unb }}$ (c). Right panels: horizontal kinetic energy, total (d), quasi-geostrophic (e) and unbalanced (f). The dispersion relation $\omega^{2}=f^{2}+f^{2} r_{n}^{2} k_{h}^{2}$ for the first 10 vertical modes is indicated by black curves. Note that the colourbar of panel (f) differs from that of panels (d) and (e).

frequency increases. This provides some information about the spontaneous-generation mechanism which we discuss further in $\S 5$.

The horizontal wavenumber-frequency spectra of the horizontal kinetic energy plotted in Figs. 4d-f indicate that the inversion (3.5) is less efficient to recover the unbalanced component of the horizontal velocity than the omega equation for the vertical velocity. 

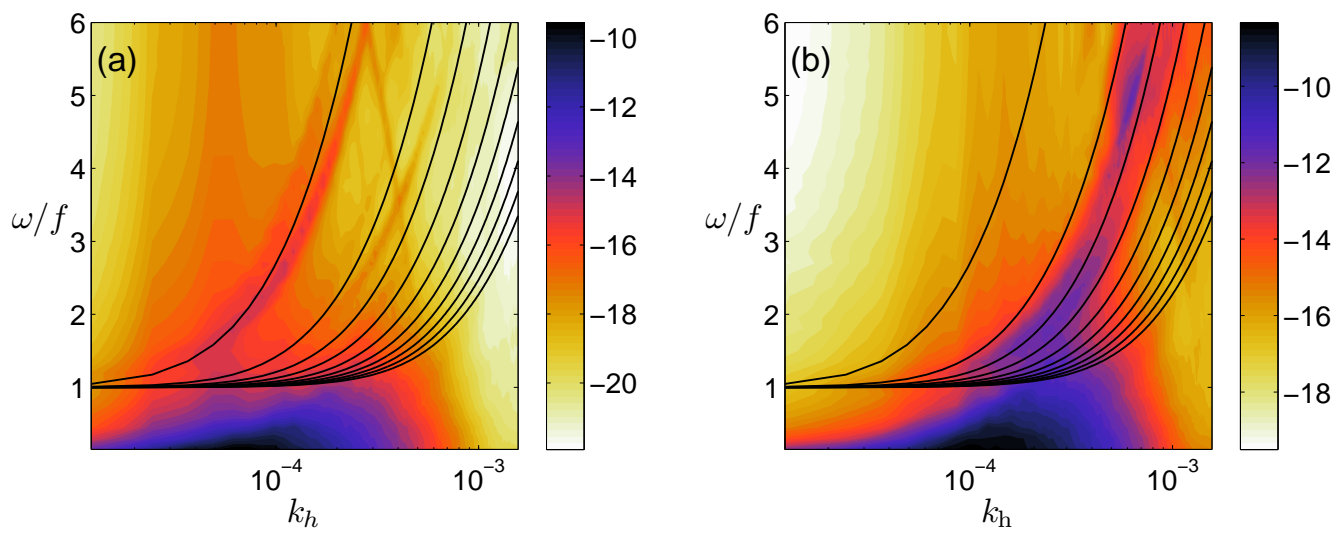

FigURE 5. Frequency-horizontal wavenumber spectrum (log-scale) of the first (a) and third (b) modes of the vertical velocity. The dispersion relation $\omega^{2}=f^{2}+f^{2} r_{d}^{2} k_{h}^{2}$ for the first 10 vertical modes is represented by the black curves. Note that the apparent reflection at the intersection of the dispersion curve for $n=1$ with the upper boundary of panel (a) results from an aliasing effect that could be eliminated by improved sampling.

Fig. 4f, in particular, shows only a weak concentration of the energy associated with $\mathbf{u}-\mathbf{u}_{\mathrm{g}}$ along the dispersion curve. This energy decreases rapidly as frequency and wavenumber increase, unlike the vertical-velocity spectrum in Fig. 4c; this is consistent with the polarisation relations (3.10), which predicts a decrease of the kinetic energy $\left|\hat{u}_{n}\right|^{2}+\left|\hat{v}_{n}\right|^{2}$ in $k_{\mathrm{h}}^{-2}$ as frequency and wavenumber increase for a constant $\left|\hat{w}_{n}\right|^{2}$. In any case, most of the energy associated with $\mathbf{u}-\mathbf{u}_{\mathrm{g}}$ is at frequencies lower than $f$ and large scales, indicating that the inversion method (3.5) is not sufficiently accurate to separate IGWs from the balanced part of the flow. The omega equation performs substantially better, unsurprisingly since its error is $O\left(\mathrm{Ro}^{2}\right)$ while that of the inversion (3.5) is only $O(\mathrm{Ro})$.

\subsection{IGW energy}

Even though the amplitudes of IGWs excited by spontaneous generation are very small, it is of interest to estimate the energy $\mathcal{E}$ and vertical flux of energy $\overline{p_{\mathrm{unb}} w_{\mathrm{unb}}}$ for the IGW field in our simulation. Because of the limitations of the inversion (3.5) for the small wave amplitudes we have to deal with, it is not possible to obtain a reliable estimate of the vertical energy flux. We therefore concentrate on the energy itself and use (3.11)-(3.12) to infer the vertical profile of the IGW energy density $\mathcal{E}_{z}$ and total IGW energy $\mathcal{E}$ from $w_{\text {unb }}$ approximated as $w-w_{\mathrm{qg}}$.

A first step is to compute the horizontal wavenumber-frequency spectrum of each mode $n$. Figs. 5a-b display the spectra of modes 1 and 3 respectively. Both spectra clearly show a concentration of energy along the dispersion relation of the mode considered, with an almost-constant amplitude as Fig. 4e already suggested. (Note that some mode-1 energy is localised near the dispersion relation of mode 2 ; this is unsurprising since the hypothesis of free linear IGWs propagating in a fluid at rest is not satisfied exactly.) The energy at $\omega \lesssim f$ apparent in Figs. 5a-b is associated with the part of the balanced velocity not resolved by the omega equation and cannot be attributed to IGW motion. Note that this effect is much stronger when separate vertical modes are considered than for the full vertical velocity at $z=-2500 \mathrm{~m}$ (see Fig. 4e); this is because the calculation of a component $n$ according to

$$
w_{n}=\frac{1}{f^{2} r_{n}^{2}} \int_{-H}^{0} N^{2}\left(w_{\mathrm{unb}}+O\left(\mathrm{Ro}^{2}\right)\right) H_{n} d z
$$



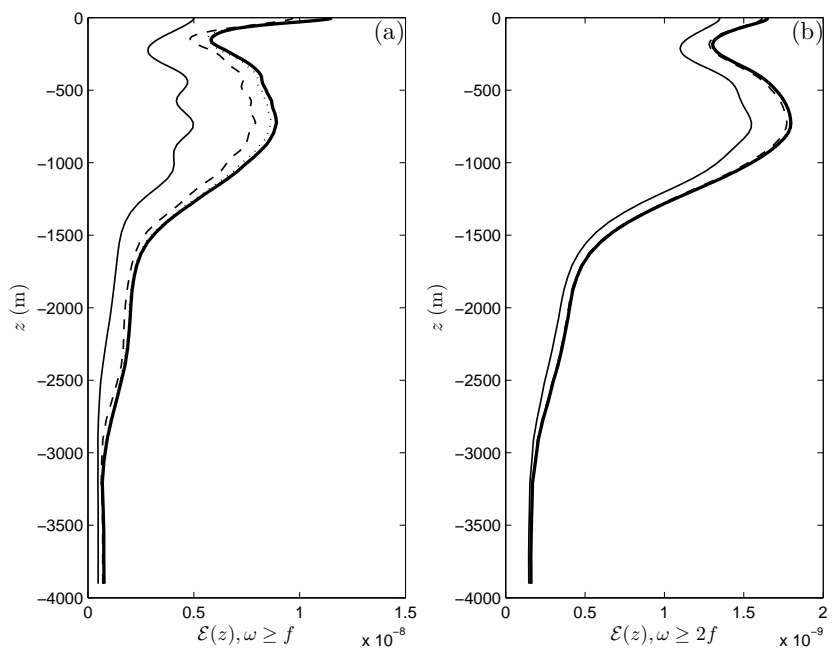

Figure 6. Vertical density of the IGW energy: the contributions of the first 5 (thin solid line), 10 (dashed line), 15 (dotted line) or 20 (thick solid line) vertical modes are shown for frequencies higher than $f$ (a) or $2 f(\mathrm{~b})$. The profiles are averaged over the duration of the simulation (see equation 3.11). Note the different scales in (a) and (b).

involves an integral over the whole depth of the domain, including surface regions where the $O\left(\mathrm{Ro}^{2}\right)$ error terms are important (since Ro can be large locally) and where $N^{2}$ is large. (Typical profiles of the functions $H_{n}$ can be found in Fig. 1c in Danioux et al. (2008).) In order to estimate the IGW energy as accurately as possible, we have therefore restricted the domains of integration in (3.11) and (3.12) to regions around the dispersion relation of each mode: for example, for mode 3 , the energy located beyond the dispersion relations of neighbouring modes 2 and 4 is removed.

The vertical profile of energy $\mathcal{E}_{z}$ is plotted in Fig. $6 \mathrm{a}$, where we restricted our calculation to the first 20 vertical modes (thick black line). The figure also displays the cumulative contribution of the first 5, 10 and 15 modes and shows that most of the energy is contained in modes $1-10$, with a substantial contribution of relatively high modes $(5<n \leq 10)$. Also represented in Fig. 6b is the energy profile associated with the IGWs of frequency higher than $2 f$; as expected, for these waves, the convergence is faster with more than $98 \%$ of the energy being in modes $\leq 10$. The energy profile of Fig. 6a displays similarities with the profile of $N$ shown in Fig. 1. This can be understood in the context of the geometric-optics (or WKB) approximation, which applies strictly to high modes only but qualitatively to all but the gravest modes $n=1,2,3$. In this approximation and ignoring Doppler-shift effects, the dispersion relation

$$
\omega^{2}=f^{2}+N^{2} \frac{k^{2}+l^{2}}{m^{2}},
$$

where $m$ is the (local) vertical wavenumber, applies (e.g. Gill 1982, chap.8), and the energy density, $\mathcal{E}_{x y z}$ say, is conserved:

$$
\frac{\partial \mathcal{E}_{x y z}}{\partial t}+\nabla \cdot\left(\boldsymbol{c}_{g} \mathcal{E}_{x y z}\right)=0
$$

where $\boldsymbol{c}_{g}=\left(c_{g x}, c_{g y}, c_{g z}\right)$ is the group velocity (e.g. Bühler 2009, p.83). Averaging over time and the horizontal coordinates, this reduces to

$$
\frac{\partial}{\partial z}\left(\mathcal{E}_{z} c_{g z}\right)=0
$$


Since, according to (4.1),

$$
c_{g z}=\frac{\partial \omega}{\partial m}= \pm \frac{\left(\omega^{2}-f^{2}\right)^{3 / 2}}{\omega k} \times \frac{1}{N(z)},
$$

where the first factor is independent of $z$, it follows that $\mathcal{E}_{z} \propto N(z)$ for high modes.

Integrating the profile $\mathcal{E}_{z}$ in Fig. 6 over the depth of the domain, we find the average energy per volume unit of the IGWs generated spontaneously in our simulation to be $\mathcal{E}=1 \cdot 10^{-5} \mathrm{~J} \mathrm{~m}^{-3}$. To put this into perspective, we can compare this result with the energy of the oceanic IGW field as described by the empirical Garrett-Munk spectrum. This yields the much larger estimate of $\mathcal{E}=1 \mathrm{~J} \mathrm{~m}^{-3}$ (e.g. Thorpe 2005). Thus, the source of IGWs provided by spontaneous generation appear to be orders of magnitude weaker than the main sources namely generation by wind and by tides and their interactions with topography. Although the energy of IGWs in our simulation is probably diminished by the artificially large dissipation of the numerical model, this conclusion is certainly robust; it is also confirmed by comparison with the energy of wind-generated inertial waves in a model with similar configuration (compare Fig. 3 with Fig. 1d in Danioux et al. (2011)).

It should be remarked that the hydrostatic approximation, employed for the numerical model as well as for the analysis leading to (3.9) and (4.1) and which assumes IGW frequencies significantly smaller than $N$, is invalidated near the bottom where $N \approx f$. The approximation is however well justified near the surface where the spontaneous emission occurs. In addition, it holds for waves with frequencies $\omega \lesssim 2 f$, which contain most of the energy (see Fig. 6), for depth down to $3000 \mathrm{~m}$ so that the vertical propagation process is also largely described faithfully.

\subsection{Generation and evolution of a wavepacket}

In this section, we examine the generation and early stage of development of a specific IGW packet. It is typically difficult to extract information on the structure of IGWs, as the motion at a given point is the sum of different wavepackets generated at different times/places which have propagated. However, immediately after the generation of a wavepacket, the associated vertical velocities are sufficiently large that they dominate the background of interfering waves. Thus the sites and times of generation can be identified easily by large values of the local r.m.s. of $w$. In Fig. 3c, for example, the region around $(x, y)=(210 \mathrm{~km}, 1150 \mathrm{~km})$ is such a generation site.

Fig. 7 focuses on the generation of a particular wavepacket by juxtaposing plots of the unbalanced vertical velocity at $z=-2500 \mathrm{~m}$ and every 12 hours with the corresponding plots of the surface relative vorticity. The vertical velocity plots (left panels) clearly show the generation of an IGW wavepacket which emerges from the background field. Its amplitude gradually increases until it reaches values about 10 times larger than the background values in Fig. 7g. In the subsequent evolution (not shown), the wavepacket propagates and disperses, and its amplitude decreases slowly to the background value. The generation of the wavepacket coincides in time and space with the appearance of a strong relative vorticity filament at the surface (Figs. $7 \mathrm{~b}, \mathrm{~d}, \mathrm{f}, \mathrm{h}$ ), with values of vorticity reaching $3 \cdot 10^{-4} \mathrm{~s}^{-1}$. The increase in vorticity results from the straining of a filament of surface density, a process well understood in the SQG approximation (e.g. Held et al. 1995). A significant feature in the evolution of the vorticity is that it is more complex than the simple straining of a front or even a straight filament, with filament curvature and displacement of the vorticity maximum expected to play a role in the process of spontaneous generation. The spatial scale of both the filament and the wavepacket that

emanates from it is quite close to the model resolution but these features are nonetheless 

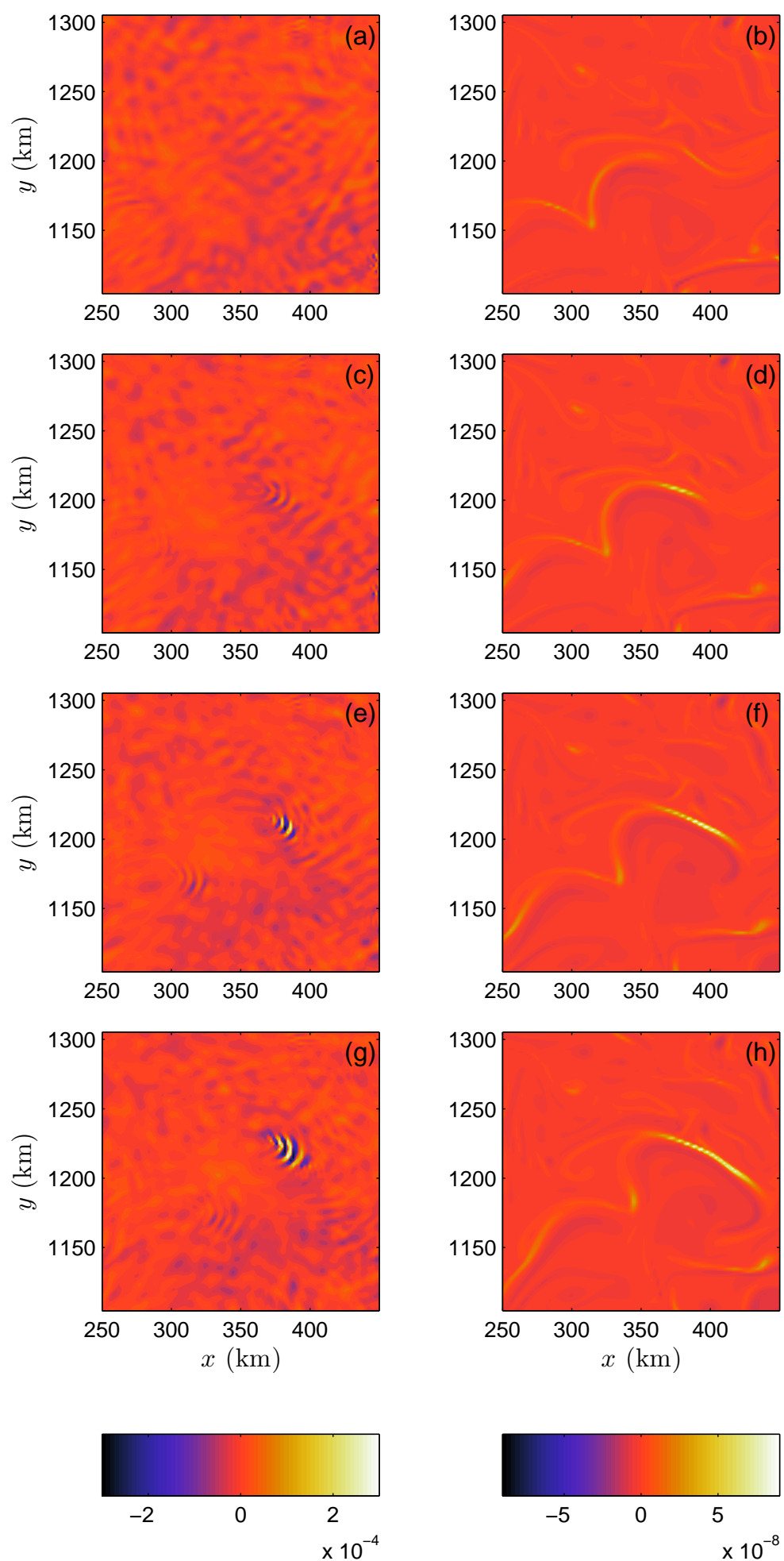

FIGURE 7 . Generation of a wavepacket by a vorticity filament. Left panels: unbalanced vertical velocity $w_{\text {unb }}$ at $z=-2500 \mathrm{~m}$. Right panels: $\operatorname{sign}(\zeta) \cdot \zeta^{2}$, where $\zeta$ is the surface relative vorticity, at different instants separated by $12(\mathrm{c}, \mathrm{d}), 24(\mathrm{e}, \mathrm{f})$ and $36(\mathrm{~g}, \mathrm{~h})$ hours from panels $(\mathrm{a}, \mathrm{b})$. 
well resolved, and we expect they would remain mostly unchanged in simulations with higher resolution but the same level of dissipation. The situation might be different for higher-resolution simulations with significantly lower dissipation, however; we comment further on this point in $\S 5$.

We have checked in our simulation that the generation scenario just described is typical of all wavepacket generation events observed. This demonstrates that spontaneous IGW generation is intimately linked to the dynamics of the surface: more precisely, the generation sites are associated with strong stretching events, which lead to large values of the surface relative vorticity, with the local Rossby number reaching values of 3 to 4. Below these values, little or no wavepacket generation appear to happen. The strong sensitivity to the local Rossby number is consistent with theoretical models predicting IGW amplitudes depending exponentially on $\mathrm{Ro}^{-1}$ (Vanneste \& Yavneh 2004; Ólafsdóttir et al. 2008; Vanneste 2008b). As a result, spontaneous generation is a highly localised and intermittent source of IGW activity.

A more detailed picture of the propagation of wavepackets is provided by the vertical structure of the unbalanced vertical velocity. Although the omega equation best extracts the unbalanced vertical velocity at depth (because of a better ratio of unbalanced to balanced vertical velocities), it is sometimes possible to identify the waves up to the surface during or immediately after a generation event thanks to their comparatively strong amplitude. Fig. 8 displays vertical (left panels) and horizontal sections (the middle panels in subsurface; the right panels at depth) of the unbalanced vertical velocity during and after the generation of a wavepacket at four instants separated by 7.5-hour intervals. The phase lines associated with the wavepacket are identified by the alternating stripes. The large amplitude patches of approximately $10 \mathrm{~km}$-scale concentrated near the surface in the left and middle panels are submesoscale features poorly filtered out by our method of extraction of the unbalanced signal: recall that what is represented here is the sum of the IGW vertical velocity plus a balanced residual of order $O\left(\mathrm{Ro}^{2}\right)$ (see §3.1): at surface this residual can become large.

Fig. 8 indicates northward propagation (i.e., to right on the figure) of the wavepacket energy, corresponding to a group velocity $\boldsymbol{c}_{g}=\left(c_{g x}, c_{g y}, c_{g z}\right)$ with $c_{g y}>0$. This, along with the slope of the phase lines, indicates downward propagation of the wave energy, that is, $c_{g z}<0$ (e.g. Gill 1982). This is consistent with our interpretation as IGWs spontaneously generated by dynamics near the surface. This is also confirmed by the fact that the wavepacket appears at $z=-200 \mathrm{~m}$ before $z=-2500 \mathrm{~m}$ (compare Figs. 8b1 and $\mathrm{c} 1$, and later on $\mathrm{b} 2$ and $\mathrm{c} 2$ ). The phase and group velocity vectors are indicated in Fig. 8a2. Values for the horizontal and vertical wavenumbers near the thermocline are $l=8 \cdot 10^{-4} \mathrm{~m}^{-1}$ and $m=1.2 \cdot 10^{-2} \mathrm{~m}^{-1}$ respectively.

Another feature of the wavepacket is the decrease of the vertical wavenumber with depth, reaching a near-zero value between $z=-2000 \mathrm{~m}$ and $z=-4000 \mathrm{~m}$. This can be understood using the geometric-optics for wavepackets in a variable medium (e.g. Bühler 2009, p.74). At short times after the wavepacket generation, advection by the balanced flow can be neglected, so that the horizontal wavenumber remains constant following the wavepacket. This can be verified from Fig. 8 which shows that the horizontal wavenumber in subsurface is approximately the same as in depth. This property, along with the conservation of the frequency $\omega$ in (4.1), implies that the ratio $N / m$ is kept constant as the wavepacket propagates downwards. This argument predicts a vertical wavenumber at $z=-3000 \mathrm{~m}$

$$
m(-3000 \mathrm{~m})=\frac{N(-3000 \mathrm{~m})}{N(-600 \mathrm{~m})} \times m(-600 \mathrm{~m}) \simeq 1.9 .10^{-3} \mathrm{~m}^{-1},
$$



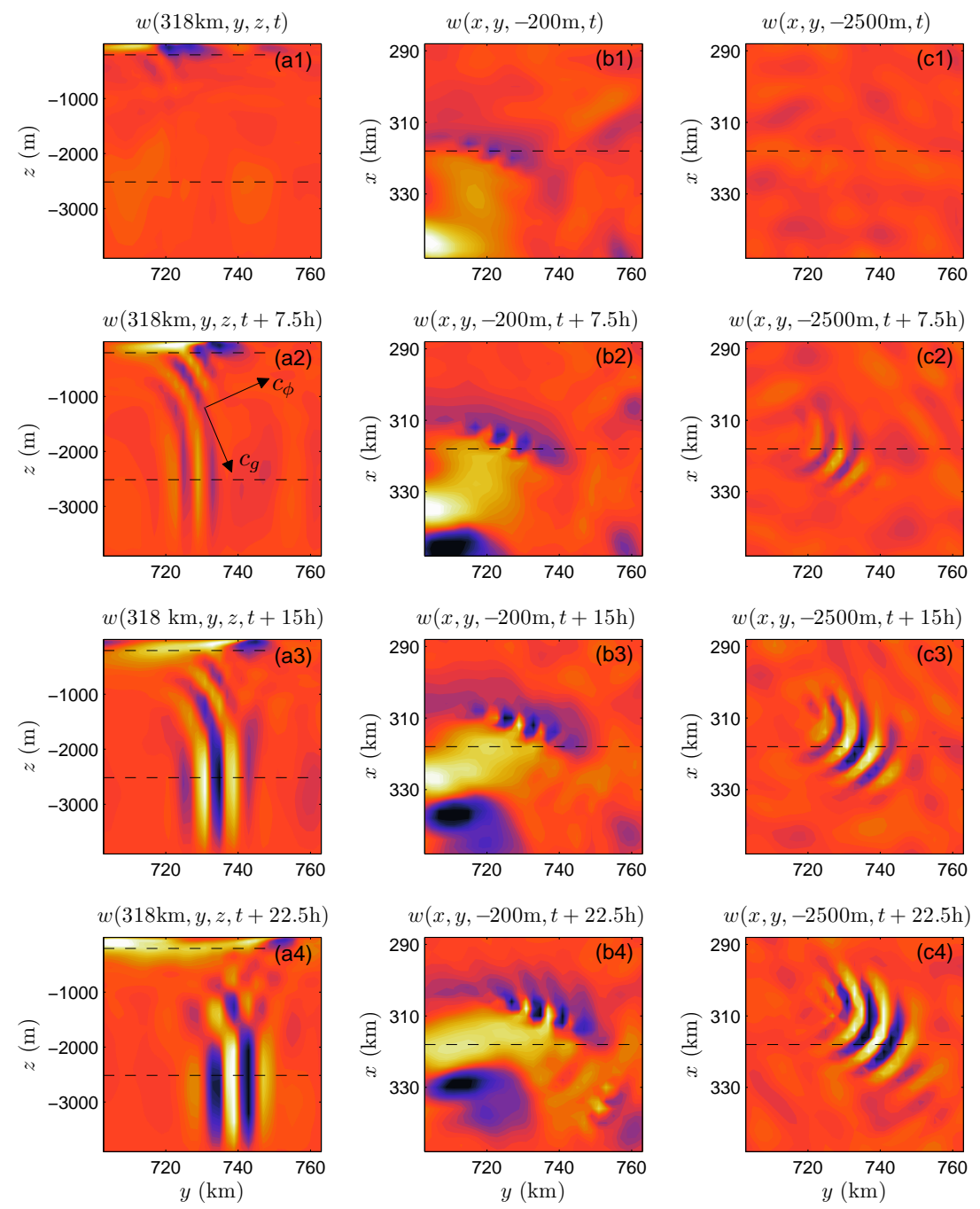

Figure 8. Time evolution of a wavepacket. The difference between the total and quasigeostrophic vertical velocities $w-w_{q g}=w_{\text {unb }}+0\left(\mathrm{Ro}^{2}\right)$ is shown in vertical (left column) and horizontal (middle and right columns) cross sections at different instants separated by 7.5 (a2,b2,c2), 15 (a3,b3,c3) and 22.5 (a4,b4,c4) hours from panels (a1,b1,c1). The horizontal cross sections correspond to $z=-200 \mathrm{~m}$ (middle column) and $-2500 \mathrm{~m}$ (right column). These sections are indicated on the left panels by the dashed lines. The vertical cross section corresponds to $x=318 \mathrm{~km}$, as indicated by the dashed line in the middle and right panels. The colourbar extends from -4 to $4 \cdot 10^{-4} \mathrm{~m} \mathrm{~s}^{-1}$. Note that absolute values beyond the range of the colourbar may occur locally.

which corresponds to a wavelength of the order of the depth of the domain, hence the almost vertically homogeneous waves in depth as observed. Note that a slight reflection on the bottom surface is visible in Fig. $8 \mathrm{a} 4$ in the form of phase lines with slope opposite to that of the main wavepacket.

At large times, the advection by the balanced flow cannot be neglected: the wavepacket propagates and is deflected by the flow. The wavenumber can change as a result of 
advection, explaining why, as observed in Fig. 4e, there is a broad range of horizontal scales in the IGW field, while the scales at generation are rarely larger than 5-10 km.

\section{Discussion}

The analysis reported in this paper demonstrates that the evolution of near-surface turbulence, which at large scales at least is well modelled by the SQG theory, is accompanied by the emission of IGWs propagating into the fluid interior. This emission is an example of spontaneous generation of IGWs by time-dependent balanced flows, a phenomenon that is generic to all flows described by the primitive equations. What makes surface motion particulary interesting, however, is that the associated scale cascade leads naturally to large Rossby number at small scales (Juckes 1994) and, as a result, to increased IGW generation. Because this generation occurs at small scales, their simulation in a flow that is forced at large scales only requires a very high resolution.

In the simulation described here, IGW generation is found to be highly localised in space and time. This is consistent with the exponential dependence in the Rossby number that is predicted for idealised models using asymptotic methods (Vanneste 2008b, and references therein): the exponential dependence is extremely sharp and implies that a relatively smooth spatial distribution of local Rossby number corresponds to highly intermittent wave generation. The small-Rossby-number asymptotic results are only indicative, however, since the IGW generation events identified in the simulation take place in regions where the Rossby number is well above 1 . It is in fact difficult to see how wave generation in this regime can be analysed by means other than direct simulations: at the generation sites, there is no separation of either time or spatial scales between balanced and unbalanced motions.

Nonetheless, because the balanced motion is localised near the surface, the Rossby numbers are small in the fluid's interior, and the separation between balanced and unbalanced motions is well justified there. This is why we are able to isolate IGWs successfully at depth using nothing more sophisticated than the quasi-geostrophic relations to approximate the balanced motion. One of the interesting features that emerge from the analysis is that the IGW vertical-velocity spectrum is approximately constant along the branches of the IGW dispersion relation in the $\left(k_{\mathrm{h}}, \omega\right)$-plane. This corresponds to a total IGW energy that decays like $k_{\mathrm{h}}^{-2}$ or, equivalently, like $\left(\omega^{2}-f^{2}\right)^{-1}$. It is tempting to relate the $k_{\mathrm{h}}^{-2}$ behaviour to the energy spectrum $E(k) \sim k_{\mathrm{h}}^{-2}$ of SQG-like turbulence, but this may be misleading since strength of the balanced motion as a source of IGWs is unlikely to depend specifically on the balanced-motion energy. For example, it may be argued that it is the matching between the frequencies of the balanced motion and those of gravity waves that matters; in this case, the frequency spectrum of the balanced motion $\tilde{E}(\omega)=E\left(k_{\mathrm{h}}\right) \mathrm{d} k_{\mathrm{h}} / \mathrm{d} \omega$ is relevant. Arguments based on WKB theory in fact suggest that the Lagrangian frequency spectrum should be considered (Aspden \& Vanneste 2010).

The amplitudes of the IGWs generated spontaneously in the simulation analysed here are very small when compared to typical amplitudes of oceanic IGWs: indeed, their energy is found to be smaller by a factor of about $10^{5}$ than the energy inferred from the Garrett-Munk spectrum. It can be argued that the limited resolution of the numerical model reduces substantially the efficiency of spontaneous generation. In SQG-like turbulence, frequencies increase as spatial scales decrease, so that the Rossby numbers, and hence presumably IGW generation, are the largest at the smallest scales. Submesoscale instabilities as described in Capet et al. (2008b), which are smoothed in our simulation, are also likely to play a significant role for spontaneous generation. However, in view of 
the comparatively much larger amplitudes of IGWs generated by other processes (wind, tides), we have to conclude that spontaneous generation is unlikely to be a significant contributor to the total IGW energy in the ocean. A similar conclusion cannot be drawn for the atmosphere, however, simply because, there are in this case no clear alternative sources of non-stationary IGWs.

This research was supported by grant NE/F002807/1 of the UK Natural Environment Research Council.

\section{REFERENCES}

AdAms, J. 1989 Mudpack: Multigrid fortran software for the efficient solution of linear elliptic partial differential equations. Applied Math. and Comput. 34, 113-146.

Aspden, J. M. \& VAnneste, J. 2010 Inertia-gravity-wave generation: a geometric-optic approach. In IUTAM Symposium on Turbulence in the Atmosphere and Oceans (ed. D. Dritschel).

Bender, C. M. \& Orszag, S. A. 1999 Advanced mathematical methods for scientists and engineers. Springer.

Blumen, W. 1978 Uniform potential vorticity flow. Part I: theory of wave interactions and two-dimensional turbulence. J. Atmos. Sc. 35, 421-432.

BüHler, O. 2009 Waves and mean flows. Cambridge University Press p. 341pp.

Capet, X., McWilliams, J. C., Molemaker, M. J. \& Shchepetkin, A. F. 2008 a Mesoscale to submesoscale transition in the california current system. Part I: Flow structure, eddy flux, and observational tests. J. Phys. Oceanogr. 38, 29-43.

Capet, X., McWilliams, J. C., Molemaker, M. J. \& Shchepetkin, A. F. 2008 b Mesoscale to submesoscale transition in the california current system. Part II: Frontal processes. $J$. Phys. Oceanogr. 38, 44-64.

Capet, X., McWilliams, J. C., Molemaker, M. J. \& Shchepetkin, A. F. 2008c Mesoscale to submesoscale transition in the california current system. Part III: Energy balance and flux. J. Phys. Oceanogr. 38, 2256-2269.

DAnioux, E. \& KLein, P. 2008 A resonance mechanism leading to wind-forced motions with a $2 f$ frequency. J. Phys. Oceanogr. 38, 2322-2329.

Danioux, E., Klein, P., Hecht, M., Komori, N., Roullet, G. \& Gentil, S. L. 2011 Emergence of wind-driven near-inertial waves in the deep ocean triggered by small-scale eddy vorticity structures. J. Phys. Oceanogr. 41, 1297-1307.

Danioux, E., Klein, P. \& Rivière, P. 2008 Propagation of wind energy into the deep ocean through a fully turbulent mesoscale eddy field. J. Phys. Oceanogr. 38, 2224-2241.

Gill, A. 1982 Atmosphere ocean dynamics. Academic Press, New York p. 662pp.

Griffiths, M. \& ReEder, M. J. 1996 Stratospheric inertia-gravity waves generated in a numerical model of frontogenesis. I: Model solutions. Q.J.R. Meteorol. Soc. 122, 1153-1174.

Held, I., Pierrehumbert, R., Garner, S. \& Swanson, K. 1995 Surface quasi-geostrophic dynamics. J. Fluid Mech. 282, 1-20.

Hoskins, B., Draghici, I. \& Davies, H. 1978 A new look at the $\omega$-equation. Q. J. R. Meteorol. Soc. 104, 31-38.

Hoskins, B. J. \& Bretherton, F. P. 1972 Atmospheric frontogenesis models: mathematical formulation and solutions. J. Atmos. Sc. 29, 11-37.

Juckes, M. 1994 Quasi-geostrophic dynamics of the tropopause. J. Atmos. Sc. 51, 2756-2768.

Karsten, R., Jones, H. \& Marshall, J. 2002 The role of eddy transfer in setting the stratification and transport of a circumpolar current. J. Phys. Oceanogr. 32, 39-54.

Klein, P., Hua, B., Lapeyre, G., Capet, X., Gentil, S. L. \& Sasaki, H. 2008 Upper ocean turbulence from high-resolution 3d simulations. J. Phys. Oceanogr. 38, 1748-1763.

Kraichnan, R. H. 1971 Inertial-range transfer in two- and three-dimensional turbulence. $J$. Fluid Mech. 47, 525-535.

Lapeyre, G. \& Klein, P. 2006 Dynamics of the upper oceanic layers in terms of surface quasigeostrophy theory. J. Phys. Oceanogr. 36, 165-176. 
Large, W., Doney, S. \& McWilliams, J. 1994 Oceanic vertical mixing: A review and a model with a nonlocal boundary layer parameterization. Rev. Geophys. 31, 363-403.

McWilliams, J. C., Molemaker, M. J. \& Ólafsdóttir, E. I. 2009 Linear fluctuation growth during frontogenesis. J. Phys. Oceanogr. 39, 3111-3129.

Molemaker, M. J., McWilliams, J. C. \& Capet, X. 2010 Balanced and unbalanced routes to dissipation in an equilibrated eady flow. J. Fluid Mech. 654, 35-63.

Muraki, D. J., Snyder, C. \& Rotunno, R. 1999 The next order corrections to quasigeostrophic theory. J. Atmos. Sc. 56, 1547-1560.

NAgAi, T., TANDOn, A. \& Rudnick, D. 2006 Two-dimensional ageostrophic secondary circulation at ocean fronts due to vertical mixing and large-scale deformation. J. Geophys. Res. 111, C09038.

Ólafsdóttir, E. I., Olde DaAlhuis, A. B. \& VAnneste, J. 2008 Inertia-gravity-wave radiation by a sheared vortex. J. Fluid Mech. 596, 169-189.

O'Sullivan, D. \& Dunkerton, T. J. 1995 Generation of inertia-gravity waves in a simulated life-cycle of baroclinic instability. J. Atmos. Sc. 52, 3695-3716.

Plougonven, R. \& Snyder, C. 2005 Gravity waves excited by jets: propagation versus generation. Geophys. Res. Lett. 32, L18802.

Plougonven, R. \& Snyder, C. 2007 Inertia-gravity waves spontaneously excited by jets and fronts. Part I: different baroclinic life cycles. J. Atmos. Sc. 64, 2502-2520.

Reeder, M. J. \& GRIfFiths, M. 1996 Stratospheric inertia-gravity waves generated in a numerical model of frontogenesis. Part II: Wave sources, generation mechanisms and momentum fluxes. Q.J.R. Meteorol. Soc. 122, 1175-1195.

Rivière, P., TrÉguier, A.-M. \& Klein, P. 2004 Effects of bottom friction on nonlinear equilibration of an oceanic baroclinic jet. J. Phys. Oceanogr. 34, 416-432.

Shchepetkin, A. \& McWilliams, J. 2005 The regional oceanic modeling system (roms): A split-explicit, free-surface, topography-following- coordinate ocean model. Ocean Modell. 9, 347-404.

Snyder, C., Muraki, D., Plougonven, R. \& Zhang, F. 2007 Inertia-gravity waves generated within a dipole vortex. J. Atmos. Sc. 64, 4417-4431.

Snyder, C., Skamarock, W. \& Rotunno, R. 1993 Frontal dynamics near and following frontal collapse. J. Atmos. Sc. 50, 3194-3211.

Temam, R. \& Wirosoetisno, D. 2011 Slow manifolds and invariant sets of the primitive equations. J. Atmos. Sci. 68, 675-682.

Thorpe, S. A. 2005 The turbulent ocean. Cambridge University Press.

VAnneste, J. 2008 a Asymptotics of a slow manifold. SIAM J. Appl. Dynam. Syst. 7, 1163-1190.

VANNESTE, J. $2008 b$ Exponential smallness of inertia-gravity-wave generation at small Rossby number. J. Atmos. Sci. 65, 1622-1637.

VANnESTE, J. \& YAVNEH, I. 2004 Exponentially small inertia-gravity waves and the breakdown of quasi-geostrophic balance. J. Atmos. Sc. 61, 211-223.

Viudez, A. \& Dritschel, D. G. 2006 Spontaneous emission of inertia-gravity wave packets by balanced geophysical flows. J. Fluid Mech. 553, 107-117.

Warn, T., Bokhove, O., Shepherd, T. G. \& Vallis, G. K. 1995 Rossby number expansions, slaving principles, and balance dynamics. Quart. J. R. Met. Soc. 121, 723-739.

Williams, R. T. 1967 Atmospheric frontogenesis: a numerical experiment. J. Atmos. Sc. 24, 627-641.

ZHANG, F. 2004 Generation of mesoscale gravity waves in upper-tropospheric jet-front systems. J. Atmos. Sc. 61, 440-457. 